\title{
Vessel Type Determined by Vessel Architectural Imaging Improves Differentiation between Early Tumor Progression and Pseudoprogression in Glioblastoma
}

\author{
(D) M. Kim, (D).E. Park, (D) K. Emblem, (D) A Bjørnerud, and (D) H.S. Kim
}

\begin{abstract}
BACKGROUND AND PURPOSE: Currently available perfusion parameters are limited in differentiating early tumor progression and pseudoprogression with no insight about vessel size and type. We aimed to investigate differences in vessel size and type between early tumor progression and pseudoprogression in posttreatment glioblastoma and to demonstrate diagnostic performance using vessel architectural imaging.
\end{abstract}

MATERIALS AND METHODS: Fifty-eight patients with enlarging contrast-enhancing masses in posttreatment glioblastomas underwent simultaneous gradient recalled-echo and spin-echo dynamic susceptibility contrast imaging. Relative CBV and vessel architectural imaging parameters, including the relative vessel size index, peak shift between gradient recalled echo and spin-echo bolus signal peaks, and arterial dominance scores using spatial dominance of arterial/venous vessel type, were calculated and compared between the 2 conditions. The area under the curve and cross-validation were performed to compare the diagnostic performance of the relative CBV, vessel architectural imaging parameters, and their combinations.

RESULTS: There were 41 patients with early tumor progression and 17 patients with pseudoprogression. Relative to pseudoprogression, early tumor progression showed a lower peak shift $(-0.02$ versus $0.33, P=.02)$ and a lower arterial dominance score (1.46 versus $2.11, P=.001)$, indicating venous dominance. Patients with early tumor progression had higher relative CBV (1.88 versus $1.38, P=$ .02 ) and a tendency toward a larger relative vessel size index ( 99.67 versus $83.17, P=.15$ ) than those with pseudoprogression. Combining arterial dominance scores and relative CBV showed significantly higher diagnostic performance (area under the curve $=$ $0.82 ; 95 \% \mathrm{Cl}, 0.70-0.94 ; P=.02$ ) than relative CBV alone (area under the curve $=0.64 ; 95 \% \mathrm{Cl}, 0.49-0.79$ ) in distinguishing early tumor progression from pseudoprogression.

CONCLUSIONS: Vessel architectural imaging significantly improved the diagnostic performance of relative CBV by demonstrating venous dominance and a tendency toward larger vessel size in early tumor progression.

ABBREVIATIONS: AUC = area under the curve; ETP = early tumor progression; CCRT = concurrent chemoradiotherapy; GRE = gradient recalled-echo; $\mathrm{PP}=$ pseudoprogression; $\mathrm{rCBV}=$ relative $\mathrm{CBV} ; \mathrm{rVSI}=$ relative vessel size index; $\mathrm{SE}=$ spin-echo; $\mathrm{VAI}=$ vessel architectural imaging

$\mathbf{P}$ seudoprogression (PP) remains a diagnostic challenge in neuro-oncology, occurring in about $20 \%$ of patients with high-grade gliomas within 12 weeks of the completion of concurrent chemoradiotherapy (CCRT). ${ }^{1}$ An accurate and timely diagnosis of PP is critical to avoid erroneous termination of successful treatment, which could negatively influence survival. However, a confirmatory scan may be required before PP can be

Received June 22, 2020; accepted after revision November 1.

From the Department of Radiology and Research Institute of Radiology (M.K., J.E.P., H.S.K.), University of Ulsan College of Medicine, Asan Medical Center, Seoul, Korea; Department of Diagnostic Physics, (K.E.) and Unit for Computational Radiology and Artificial Intelligence (A.B.), Division of Radiology and Nuclear Medicine, Oslo University Hospital, Oslo, Norway; and Department of Physics (A.B.), University of Oslo, Oslo, Norway.

This research was supported by a National Research Foundation of Korea grant funded by the Korean government (Ministry of Science, ICT and Future Planning) (grant Nos: NRF-2020R1A2B5B01001707 and NRF-2020R1A2C4001748). confidently distinguished from early tumor progression (ETP), which might delay a prompt therapeutic intervention. Both PP and ETP manifest as new or enlarging contrast-enhancing lesions on follow-up imaging after the completion of CCRT. Abnormal enhancement in PP likely results from increased permeability caused by direct damage to the blood-brain barrier and treatment-related cellular hypoxia, which results in increased expression of hypoxia-regulated molecules in surrounding cells. ${ }^{2,3}$ This

Please address correspondence to Ji Eun Park, MD, PhD, Department of Radiology and Research Institute of Radiology, University of Ulsan College of Medicine, Asan Medical Center, 88 Olympic-ro 43-gil, Songpa-Gu, Seoul 05505, South Korea; email: jieunp@gmail.com

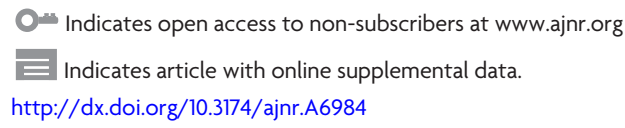

AJNR Am J Neuroradiol 42:663-70 Apr 2021 www.ajnr.org 


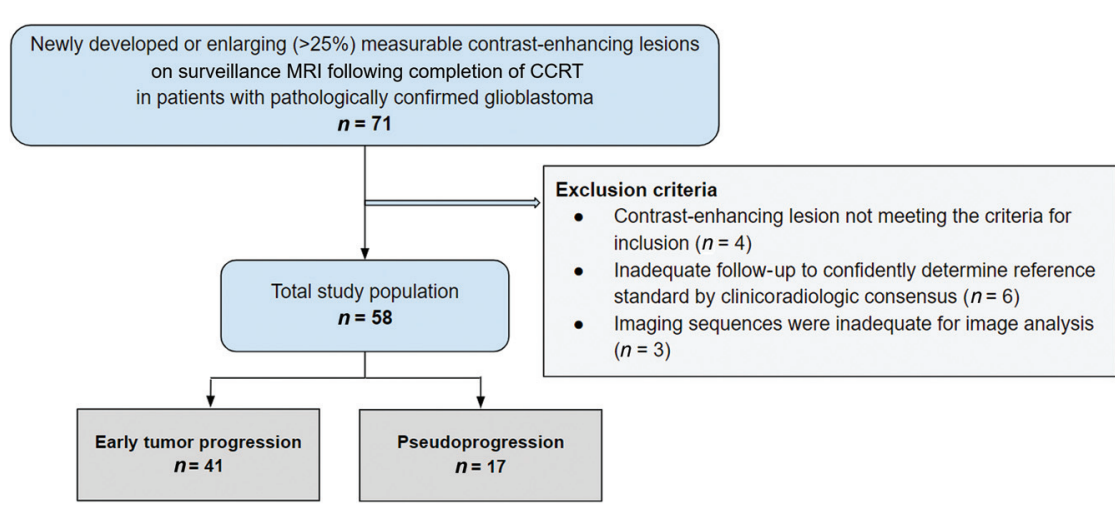

FIG 1. Flow diagram showing the patient-selection protocol and the inclusion and exclusion criteria.

appears indistinguishable from increased enhancement associated with early tumor progression, which represents tumoral neoangiogenesis. Such differences in tumoral vasculature and microenvironment cannot be shown by conventional MR imaging.

So far, perfusion parameters have shown promise in differentiating PP from ETP. CBV from DSC imaging has been found to be particularly useful for diagnosing $\mathrm{PP}^{4-7}$ Although high $\mathrm{CBV}$ on DSC imaging may result from overall increased vascular density, it does not specify whether high CBV comes from vessel recruitment or dilation of vessels. ${ }^{8}$ In addition, CBV is unable to differentiate the dominant vessel type within the ROI. Vessel architectural imaging (VAI) is a recent MR imaging technique with the potential of providing further insight into vessel size and type. Emblem et $\mathrm{al}^{9}$ demonstrated the use of VAI to characterize the vessel architecture of recurrent glioblastoma and its changes in response to antiangiogenic agents to successfully identify responders to antiangiogenic treatment. It exploits the differences in observed proton relaxation from simultaneously acquired contrast agent-enhanced gradient recalled-echo (GRE) and spin-echo (SE) MR imaging for vessel-size estimation. ${ }^{10-12}$ The temporal shift between the 2 relaxation rate curves from GRE and SE may be visualized in a hysteresis plot, and its slope and direction provide estimates of vessel size and type, respectively. It has been shown that vessel size measures on MR imaging accurately reflected histologic measures of vessel caliber while CBV correlated with vessel density in high-grade gliomas. ${ }^{8}$ Because VAI can estimate vessel size and type, it may be able to provide further insight into the mechanism of PP and to differentiate tumoral neoangiogenesis seen in ETP and treatment-induced change seen in PP.

We hypothesized that VAI could improve the diagnostic performance for identifying PP over DSC perfusion parameters by reflecting vessel size and type in the early posttreatment stage of glioblastoma. Thus, the purpose of this study was to investigate differences in vessel type and size between ETP and PP in posttreatment glioblastomas and to demonstrate the diagnostic performance in the enlarging contrast-enhancing mass using VAI.

\section{MATERIALS AND METHODS}

\section{Study Patients and Inclusion Criteria}

Asan Medical Center institutional review board approved this retrospective study, and the requirement for informed consent was waived. We searched the electronic data base of the Department of Radiology at our tertiary center and retrospectively reviewed the records of patients between August 2018 and July 2019. Figure 1 shows the patient inclusion process. We identified 71 consecutive patients who met the following inclusion criteria: 1) histopathologic diagnosis of a newly diagnosed glioblastoma according to the 2016 World Health Organization criteria; ${ }^{13}$ 2) CCRT with temozolomide from Stupp et $\mathrm{al}^{14}$ and 6 cycles of adjuvant trimetazidine performed after surgical resection or biopsy; 3) newly developed or enlarging (>25\%) and measurable contrast-enhancing lesions on surveillance MR imaging following completion of CCRT; ${ }^{15}$ and 4) DSC perfusion and VAI sequences available at the time of newly developed or enlarging and measurable contrast-enhancing lesions, and 5) pathologic diagnosis or clinicoradiologic consensus of ETP or PP. Measurable contrast-enhancing lesions were defined as bidimensionally enhancing lesions with 2 perpendicular diameters of at least $10 \mathrm{~mm}$ being visible on $\geq 2$ axial slices on conventional $\mathrm{MR}$ imaging.

A final diagnosis of PP and ETP was confirmed pathologically in second-look operations when clinically indicated. When second-look operations could not be performed, clinicoradiologic diagnoses were made by consensus between a neuro-oncologist (J.H.K. with 26 years of experience in neuro-oncology practice) and a neuroradiologist (H.S.K. with 18 years of experience in neuro-oncology imaging) according to the Response Assessment in Neuro-Oncology criteria. ${ }^{15}$ A final diagnosis of PP was made when there was an increase in contrast-enhancing lesions that subsequently regressed or became stable without any changes in the treatment for at least 6 months after an operation and completion of CCRT. Alternatively, a final diagnosis of ETP was made if enhancing lesions gradually increased on $>2$ subsequent follow-up MR imaging studies performed at 2- to 3-month intervals and required a prompt change in treatment. Patients were excluded if contrast-enhancing lesions did not meet the criteria for ETP or PP $(n=4)$, there was inadequate follow-up to confidently determine ETP or PP on clinicoradiologic consensus ( $n=$ $6)$, and VAI sequences were inadequate for image analysis $(n=$ $3)$. Finally, 58 consecutive patients were enrolled in the study.

\section{MR Imaging Protocol and Image Processing}

The brain tumor imaging protocol after completion of CCRT at our institution included T2-weighted imaging, FLAIR imaging, T1-weighted imaging, diffusion-weighted imaging, simultaneous GRE-SE DSC perfusion, contrast-enhanced T1-weighted imaging, and conventional DSC perfusion MR imaging. Simultaneous GRE-SE DSC MR imaging was acquired using axial gradientecho, spin-echo echo-planar images with $\mathrm{TR}=1.33$ seconds, $\mathrm{TE}=34$ and $103 \mathrm{~ms}$ (respectively), section thickness $=5 \mathrm{~mm}$, intersection gap $=2.5 \mathrm{~mm}$, in-plane resolution $=1.70 \times 1.70$ $\mathrm{mm}$, matrix size $=128 \times 128$, ten slices, and 120 volumes with a 

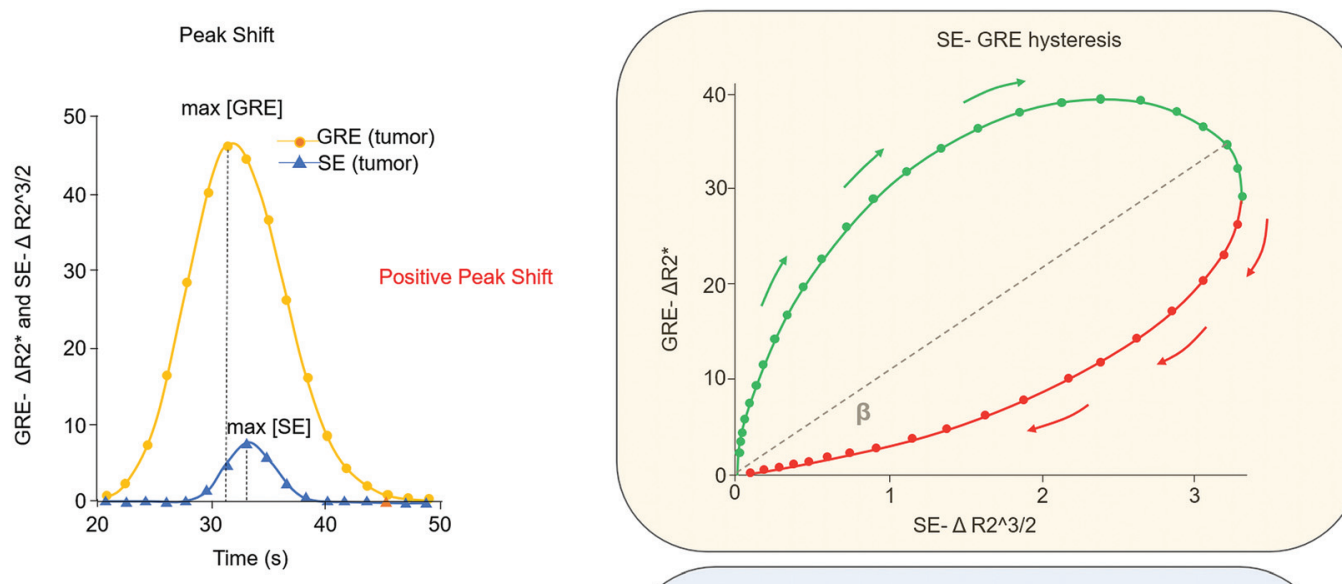

Positive vortex direction
(Clockwise)
Arterial dominancy
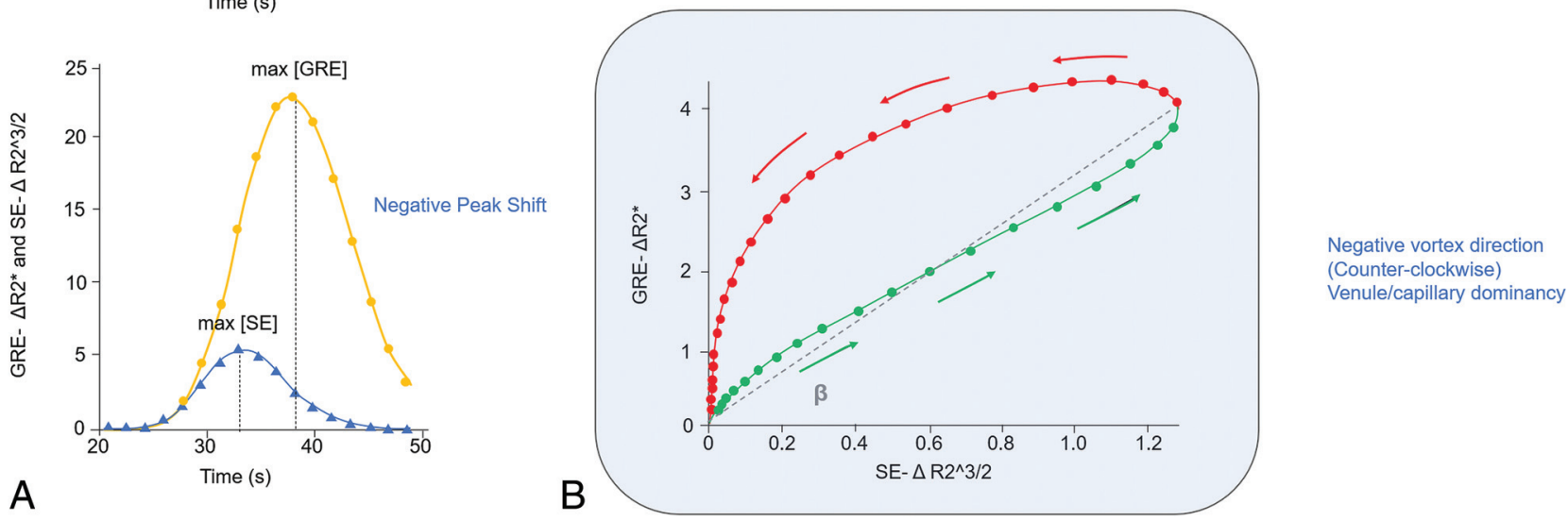

FIG 2. Peak shift and hysteresis plot. $A$, Peak shift: the time curves of GRE-based $\Delta R 2^{\star}$ and SE-based $\Delta R 2^{\wedge} 3 / 2$ were fitted to a $\gamma$-variate function. A positive peak shift indicates a GRE peak preceding the SE peak, while a negative peak shift indicates the SE peak preceding the GRE peak. $B$, Hysteresis plot: the fitted curves are plotted with $\Delta \mathrm{R} 2^{\wedge} 3 / 2$ (SE-based) along the $\mathrm{x}$-axis and $\Delta \mathrm{R} 2^{\star}$ (GRE-based) along the $y$-axis. The clockwise rotation indicates that GRE-based changes precede SE-based changes, corresponding to the positive peak shift with arterial dominance. The counter-clockwise rotation indicates that SE-based change precedes GRE-based changes, corresponding to the negative peak shift with venous dominance. Max indicates maximum.

total acquisition time of 6 minutes 30 seconds. For simultaneous GRE-SE DSC perfusion, a dynamic bolus was administered as a standard dose of $0.1 \mathrm{mmol} / \mathrm{kg}$ of gadoterate meglumine (Dotarem; Guerbet) delivered at a rate of $4 \mathrm{~mL} / \mathrm{s}$ by a MR imaging-compatible power injector (Spectris MR injector; Medrad). The bolus of contrast material was followed by a $20-\mathrm{mL}$ bolus of saline, injected at the same rate. For conventional DSC perfusion, a second, standard dose of $0.1 \mathrm{mmol} / \mathrm{kg}$ of gadoterate meglumine was administered. Conventional DSC perfusion MR imaging was acquired using $\mathrm{TR}=1.72$ seconds, $\mathrm{TE}=40 \mathrm{~ms}$, flip angle $=35^{\circ}$, FOV $=240 \mathrm{~mm}$, section thickness $=5 \mathrm{~mm}$, intersection gap $=$ $2 \mathrm{~mm}$, matrix size $=128 \times 128$, and number of slices $=20$ with a total acquisition time of 1 minute 50 seconds. The dynamic acquisition was performed with a temporal resolution of $1.5 \mathrm{sec}-$ onds, and 60 dynamics were acquired.

\section{Definition of CBV and VAI Parameters}

The simultaneous GRE-SE DSC perfusion data obtained during the first injection were used for VAI analysis, while the conventional DSC perfusion data obtained during the second injection were used for whole-brain relative $\mathrm{CBV}$ ( $\mathrm{rCBV}$ ). The wholebrain $\mathrm{rCBV}$, normalized to contralateral normal-appearing white matter, was calculated using numeric integration of the time concentration curve after correcting for contrast agent leakage. Leakage correction was performed using the method of Weisskoff et $\mathrm{al}^{16}$ with further adaptations from Boxerman et $\mathrm{al}^{17}$ for both VAI perfusion analysis and conventional DSC perfusion analysis with leakage being estimated from the deviation in each voxel according to a nonleakage reference tissue response curve.

The VAI perfusion-related maps were generated according to previously published definitions and notations. ${ }^{9,18,19}$ In short, the pixel-wise GRE and SE dynamic signals were converted to changes in $\mathrm{R} 2{ }^{*}$ and $\mathrm{R} 2$, respectively, for the GRE and SE signals. The resulting $\Delta \mathrm{R} 2$ values were then raised to the power of $3 / 2$, and a Gaussian function was fitted to the resulting time curves. The fitted curves were then plotted with $\Delta \mathrm{R} 2^{\wedge} 3 / 2$ along the $\mathrm{x}$-axis and $\Delta \mathrm{R} 2 *$ along the $y$-axis, forming hysteresis loops. The hysteresis loops were characterized by the loop direction, long axis, and slope of long axis that are shown to be affected by changes in microvascular function and structure. ${ }^{20}$ The voxelwise loop direction was visualized by the arrows representing the temporal vortex propagation (Fig 2). We estimated the following VAI metrics:

1) Relative vessel size index (rVSI): ${ }^{9}$ defined by $\mathrm{rVSI}=$ $\sqrt{r C B V \times A D C} \times \beta$, where $\beta$ is the slope of the long axis of the resulting hysteresis curve, as shown in Fig 2, ADC is the apparent diffusion coefficient, and $\mathrm{rCBV}$ is relative cerebral blood 
Table 1: Clinical characteristics of the patients ${ }^{a}$

\begin{tabular}{lccc}
\hline & ETP $(\boldsymbol{n}=41)$ & PP $(\boldsymbol{n}=17)$ & $\boldsymbol{P}$ \\
\hline Age (yr) & $57.2[\mathrm{SD}, 12.5]$ & $55.1[\mathrm{SD}, 16.0]$ & .64 \\
No. of female patients & $17(41.5 \%)$ & $11(64.7 \%)$ & .11 \\
KPS $\geq 70$ & $35(85.4 \%)$ & $14(82.4 \%)$ & .77 \\
IDH wild-type & $38(92.7 \%)$ & $15(88.2 \%)$ & .36 \\
MGMT promoter status (methylated/ $\quad 15 / 22 / 4$ & $7 / 6 / 4$ & .28 \\
$\quad$ unmethylated/NA) & & & .97 \\
Surgical extent & & & \\
$\quad$ Biopsy & $4(9.8 \%)$ & $2(11.8 \%)$ & \\
$\quad$ Partial resection & $13(31.7 \%)$ & $5(29.4 \%)$ & \\
$\quad$ Gross total resection & $24(58.5 \%)$ & $10(58.8 \%)$ & \\
Mean time interval between completion of CCRT & $204.4[\mathrm{SD}, 523.0]$ & $112.4[\mathrm{SD}, 123.9]$ & .48 \\
$\quad$ and imaging (days) & & & \\
\hline
\end{tabular}

Note:-KPS indicates Karnofsky Performance Status; IDH, isocitrate dehydrogenase; NA, not available.

${ }^{a}$ Data are expressed as the mean [SD]. Numbers in parentheses are percentages.

${ }^{b}$ Surgical extent refers to the extent of surgical resection at the initial cytoreduction surgery. and 3D contrast-enhanced T1-weighted images were coregistered to the $\mathrm{rCBV}$ and VAI maps. All image analyses were performed using the commercial software package (nordicICE, Version 4.0.6; NordicNeuroLab).

\section{Statistical Analysis}

The Student $t$ test and $\chi^{2}$ test were used to assess differences between PP and ETP groups regarding the demographic data. The Student $t$ test was used to assess differences in the rCBV and VAI parameters between the PP and ETP groups. A receiver operating characteristic curve analysis was per-

volume obtained from the GRE signal normalized to contralateral normal-appearing white matter.

2) Peak shift: the shift in the peak ( $\gamma$-fitted) GRE versus SE signals. Peak shift showed positive value when the GRE peak signal preceded the SE peak signal (Fig 2). Here, we applied a 2-stage fitting procedure for optimal stability. First, we approximated the $\gamma$ variate model parameters from linear regression after pixelwise transforming of the nonlinear $\gamma$ variate expression to a linear form, as described by Madsen. ${ }^{21}$ The estimated parameters from the linear fit were then used as initial values in a nonlinear least-squares model using a standard implementation of the iterative Levenberg-Marquardt algorithm. ${ }^{22}$ In addition, Gaussian smoothing was applied to the raw data before analysis to reduce noise.

3) Arterial dominance score: dominant vessel type (artery versus venule/capillary) was assigned for each voxel depending on the direction of the vortex. Here, arterial dominance was assigned to the voxels with a positive (clockwise) vortex direction, while venule/capillary dominance was assigned to the voxels with a negative (counterclockwise) vortex direction. Because the average value of vortex direction for a given VOI representing the contrast-enhancing mass did not account for spatial dominance of arterial-versus-venous vessel type, 2 expert radiologists (J.E.P. with 7 years of experience in neuro-oncologic imaging, and H.S.K.) determined the dominant vessel type by observation of the spatial dominance of negative (venule/capillary dominance) or positive vortex directions (arterial dominance) based on the colorized VAI vessel-type map. The arterial dominance score was assigned on the following scale: 1, capillary/venule-dominant; 2 , mixed; and 3, arterial-dominant. The readers were blinded to the clinical information and reference standard when assigning the score.

Lesion segmentation of the contrast-enhancing mass was performed using a $3 \mathrm{D}$ region-growing based on the multiplanar reconstruction view of $3 \mathrm{D}$ contrast-enhanced T1-weighted images by a neuroradiologist (M.K., with 2 years of experience in neuro-oncologic imaging) using segmentation threshold and region-growing segmentation algorithms provided by Medical Imaging Interaction Toolkit (MITK) software (www.mitk.org). All segmented images were re-evaluated and validated by an experienced neuroradiologist (H.S.K). Finally, the lesion mask formed to determine the diagnostic performance of each perfusion parameter for differentiating ETP and PP and was further validated using leave-one-out cross-validation with 100 -fold bootstrapping. The diagnostic performance of each individual VAI parameter combined with the rCBV was also evaluated. ${ }^{23}$ Subgroup analysis with patients diagnosed as ETP and PP within 9 months of completing CCRT was performed. $P$ values of areas under the curve (AUCs) from each parameter were compared using the Wald test. For the comparison of the AUCs and crossvalidation with bootstrap resampling, the pROC and cvTools packages in R, Version 3.4.3 (R Project for Statistical Computing, http://www.r-project.org) were used. A $P$ value $<.05$ was considered statistically significant.

\section{RESULTS}

\section{Patient Demographics}

The clinical characteristics of the patients are summarized in Table 1 . Of the 58 study patients, $41(70.7 \%)$ were classified as ETP, and 17 (29.3\%), as PP. Among 41 patients with ETP, 7 patients (17\%) were pathologically confirmed and 34 cases (83\%) were diagnosed as having ETP by clinic-radiologic consensus. There were no significant differences between the patients with PP and ETP in terms of age, sex, baseline Karnofsky Performance Status score, isocitrate dehydrogenase (IDH) mutation status, MGMT methylation status, extent of surgery, and mean time interval between the operation and imaging study. Subsequent to study imaging, patients were treated by reoperations or secondline agents including antiangiogenic treatment. There were no patients on experimental therapies such as vaccines or checkpoint inhibitors.

\section{Comparison of rCBV and VAI Parameters in ETP and PP}

DSC and VAI parameters are summarized in Table 2. Patients with PP showed significantly lower $\mathrm{rCBV}$ (mean $\mathrm{PP}=1.38$ [SD, 0.53 ] versus mean $\mathrm{ETP}=1.88[\mathrm{SD}, 0.78], P=.02)$ and a tendency toward a smaller rVSI $(\mathrm{PP}=83.17$ [SD, 40.60] versus $\mathrm{ETP}=99.67$ [SD, 38.78], $P=.15)$ compared with patients with ETP. Patients with PP showed a significantly higher positive peak shift compared with those with ETP $(\mathrm{PP}=0.33$ [SD, 0.77] versus $\mathrm{ETP}=-0.02$ [SD, 0.36], $P=.02$ ). The arterial dominance score was significantly higher in the PP group compared with the ETP 
Table 2: Mean perfusion and VAI parameters in ETP and PP

\begin{tabular}{lccc}
\hline \multicolumn{1}{c}{ Imaging Parameters } & ETP $(\boldsymbol{n}=\mathbf{4 1})$ & PP $(\boldsymbol{n}=17)$ & $\boldsymbol{P}_{\text {Value }}$ \\
\hline rCBV & $1.88[\mathrm{SD}, 0.78]$ & $1.38[\mathrm{SD}, 0.53]$ & .02 \\
rVSI & $99.67[\mathrm{SD}, 38.78]$ & $83.17[\mathrm{SD}, 40.60]$ & .15 \\
Peak shift & $-0.02[\mathrm{SD}, 0.36]$ & $0.33[\mathrm{SD}, 0.77]$ & .02 \\
Arterial dominance score & $1.46[\mathrm{SD}, 0.7]$ & $2.11[\mathrm{SD}, 0.6]$ & .001 \\
\hline
\end{tabular}

${ }^{a} P$ values from an independent-samples $t$ test.
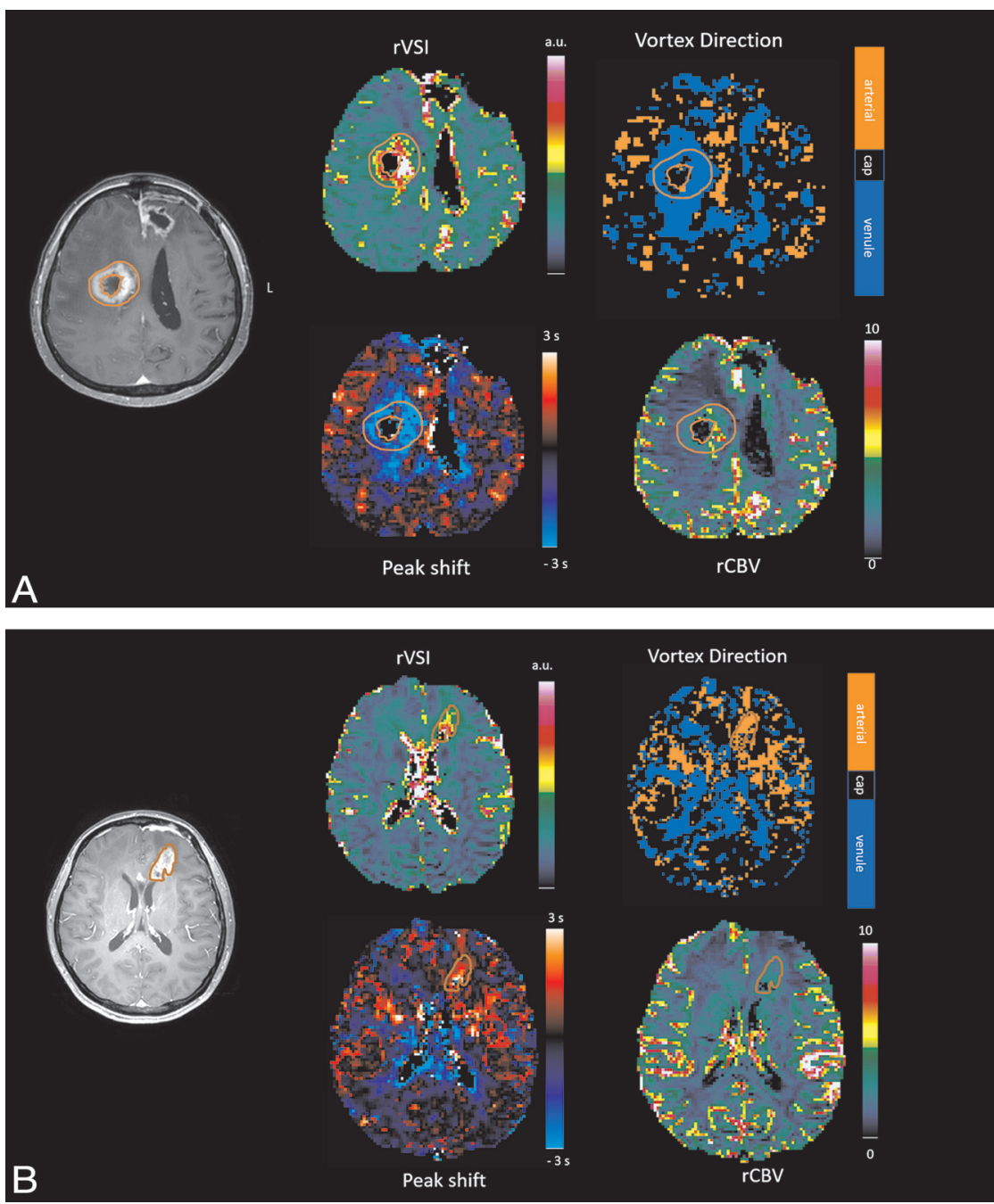

FIG 3. A, Early tumor progression. A 53-year-old woman who completed concurrent chemoradiotherapy 8 weeks ago developed a necrotic enhancing mass in the right frontal lobe surrounding the surgical cavity. On the rCBV map and rVSI map, rCBV is increased with a high rVSI in the corresponding area. A negative peak shift on the peak shift map with venous dominance on the vortex direction map is noted, and an arterial dominance score of 1 was assigned. B, Pseudoprogression. A 67-year-old woman who completed CCRT 10 weeks ago developed a necrotic enhancing mass in the left frontal lobe. On the rCBV and rVSI maps, rCBV is mildly increased with a lower rVSI than in the patient with ETP. A positive peak shift on the peak shift map and arterial dominance on the vortex direction map are shown, and an arterial dominance score of 3 was assigned.

\section{Diagnostic Performance of Individual and Combined Parameters}

Table 3 summarizes the diagnostic performance of VAI parameters when used individually and combined with $\mathrm{rCBV}$. The diagnostic performance of rCBV was 0.64 (95\% CI, 0.49-0.79) with a sensitivity of $88.2 \%$ and specificity of $48.8 \%$. Among VAI parameters, the arterial dominance score showed the highest performance with an AUC of 0.76 (95\% CI, 0.64-0.88; Wald test, $P=.005$ ), followed by peak shift (AUC $=0.60 ; 95 \%$ CI, 0.43-0.76; Wald test, $P=.20$ ) and rVSI (AUC $=$ 0.60; 95\% CI, 0.43-0.77; Wald test, $P=.58$ ).

The highest diagnostic performance was achieved when the rCBV was combined with the arterial dominance score with an AUC of 0.82 (95\% CI, $0.70-0.94)$ with a sensitivity of $82.3 \%$, specificity of $78.0 \%$, and accuracy of $79.3 \%$, which was significantly higher than $\mathrm{rCBV}$ alone $(P=.02)$. The diagnostic performance also improved when the rCBV was combined with the rVSI (AUC $=0.67 ; 95 \%$ CI, $0.53-$ $0.82)$ or peak shift $(\mathrm{AUC}=0.70 ; 95 \%$ CI, 0.56-0.86), but these did not reach statistical significance.

\section{Subgroup Analysis with Patients Diagnosed as ETP or PP within 9 Months of Completing CCRT}

There were 28 patients with ETP (68\%, 28/41) and 14 patients with PP (82\%) diagnosed within 9 months of completing CCRT. DSC and VAI parameters in patients diagnosed with ETP or PP within 9 months of completing CCRT are summarized in the Online Supplemental Data. Patients with PP showed significantly lower rCBV (mean $\mathrm{PP}=1.30[\mathrm{SD}, 0.54]$ versus $\mathrm{ETP}=1.99[\mathrm{SD}, 0.82], P=.003)$ and a higher arterial dominance score $(\mathrm{PP}=$ 2.07 [SD, 0.46] versus ETP $=1.43$ [SD, $0.68], P=.001)$. Patients with PP showed a tendency toward a higher peak shift and a smaller rVSI compared with patients with ETP.

group $(\mathrm{PP}=2.11[\mathrm{SD}, 0.6]$ versus $\mathrm{ETP}=1.46[\mathrm{SD}, 0.7], P=$ $.001)$. Figure 3 shows representative cases of PP and ETP, and corresponding signal intensity curves with hysteresis plots are provided in the Online Supplemental Data.
The diagnostic performance of VAI parameters when used individually and combined with $\mathrm{rCBV}$ is shown in the Online Supplemental Data. Among VAI parameters, the arterial dominance score showed the highest performance with an AUC of 
Table 3: Diagnostic performance of individual and combined imaging parameters from perfusion and VAI in differentiating PP from

\begin{tabular}{llccccc}
\hline \multicolumn{1}{c}{ Individual Parameters } & AUC & Criterion & 95\% CI & Sensitivity & Specificity & Accuracy \\
\hline rCBV & 0.64 & $\leq 1.74$ & $0.49-0.79$ & 88.2 & 48.8 & 60.3 \\
rVSI & 0.60 & $\leq 70.8$ & $0.43-0.77$ & 47.5 & 75.6 & 67.2 \\
Peak shift & 0.60 & $>0.345$ & $0.43-0.76$ & 29.4 & 92.6 & 74.1 \\
Arterial dominance score & 0.76 & $>1$ & $0.64-0.88$ & 88.2 & 65.8 & 72.4 \\
rCBV + rVSI & 0.67 & NA & $0.53-0.82$ & 94.1 & 39.05 & 55.2 \\
rCBV+ peak shift & 0.70 & NA & $0.56-0.86$ & 100 & 39.0 & 56.9 \\
rCBV + arterial dominance score & 0.82 & NA & $0.70-0.94$ & 82.3 & 78.0 \\
\hline
\end{tabular}

Note:-NA indicates not available.

0.78 (95\% CI, 0.64-0.91). The highest diagnostic performance was achieved when the $\mathrm{rCBV}$ was combined with the arterial dominance score with an AUC of 0.86 (95\% CI, 0.75-0.98).

\section{DISCUSSION}

In this study, we demonstrated that vessel architectural imaging parameters derived from simultaneously acquired contrast agent-enhanced gradient-echo and spin-echo MR imaging improved the diagnostic performance of $\mathrm{rCBV}$ by determining vessel size and type in differentiating early tumor progression from pseudoprogression. Early tumor progression showed venous dominance and a tendency toward larger vessel size, while pseudoprogression showed arterial dominance and a tendency toward smaller vessel size. The spatial dominance of vessel type was analyzed using the arterial dominance score, which was lower in early tumor progression. VAI parameters were able to provide insight into tumor vasculature and mechanism of pseudoprogression by providing information about vessel size and type and may be used to improve the diagnostic performance of $\mathrm{rCBV}$ in diagnosing tumor progression in posttreatment glioblastoma.

The GRE and SE readouts have different sensitivities to contrast agent-induced susceptibility effects, with the GRE data reflecting vessels of all sizes and SE data predominantly reflecting capillaries. ${ }^{20}$ Peak shift is mainly affected by the relationship between arterial and venous volumes, ${ }^{19}$ and negative peak shift indicates SE preceding the GRE peak with venous dominance. In our study, a negative peak shift was obtained in the ETP group, implying an SE peak preceding the GRE peak with venous dominance, which corresponds to a counterclockwise direction in the hysteresis plot. The venous dominance of the tumor vasculature may be attributed to arteriovenous connections within the tumor that lead to increased vessel size and venous flow and reduced flow in the feeding arterioles. ${ }^{24}$ Tissue with treatment-related change, on the other hand, showed a positive peak shift (GRE peak preceding the SE peak) with arterial dominance. We speculate this as relatively less venous dominance in tissue with treatment-related change with low rCBV values. In recurrent tumor, proliferation of microvessels at early neovascularization ${ }^{25}$ may be reflected in the SE peak by its high sensitivity to microvessels, while treatment-related change showed a less prominent SE peak resulting in a positive peak shift with the GRE peak preceding the SE peak.

In our study, the rVSI was not shown to be significantly different between ETP and PP, though there was a tendency toward larger vessel size in ETP. A previous study ${ }^{26}$ showed that mean vessel size was higher in tumor progression (89 [SD, 45] $\mu \mathrm{m})$ compared with contralateral normal white matter (40 [SD,
11] $\mu \mathrm{m})$ with borderline significance in a subgroup analysis with patients suspected of tumor progression within 9 months after completing CCRT. In addition, vessel size measures on MR imaging were reported to demonstrate higher specificity and sensitivity in differentiating high-grade from low-grade gliomas compared with $\mathrm{rCBV} .{ }^{8} \mathrm{VAI}$ was able to provide information regarding vessel size and type better, reflecting underlying tumor vasculature, and hence showed improved diagnostic performance in detecting tumor progression.

The vasculature of viable tumor and damaged tissue is spatially complex and heterogeneous, and conventional perfusion parameters such as CBV cannot currently reflect such spatial complexity and heterogeneity. ${ }^{26}$ The arterial dominance score in this study successfully accounted for the spatial dominance of arteries in PP over capillaries or venules. Previous studies reported vessel type using the hysteresis $\operatorname{plot}^{18,26}$ or binarized value $^{26}$ of arterial or venous dominance, but these reports were limited to selected voxels and did not account for dominance of a particular vessel type or spatial heterogeneity. The arterial dominance score was reported on a 3-number scale, and fraction of dominance was considered, which may suggest that vessel type as well as its spatial dominance can be helpful in differentiating PP from ETP in posttreatment glioblastoma. In the current study, the arterial dominance score was established by 2 experienced neuroradiologists. In future studies, reproducibility and interobserver agreement of the arterial dominance score among readers with different levels of experience may need to be evaluated to validate this parameter. Moreover, the possibility of deriving this score directly from the VAI images may be investigated to eliminate the need for manual expert analysis.

In the meta-analyses based on DSC perfusion MR imaging, pooled sensitivities and specificities for the best-performing parameters ranged from $82 \%$ to $90 \%$ and from $86 \%$ to $95 \%$, respectively. ${ }^{6,7,27}$ However, these analyses did not strictly differentiate between late radiation necrosis and early treatment effects of pseudoprogression. Our results showed that $\mathrm{rCBV}$ was a sensitive parameter (88.2\%) with low specificity (48.8\%), while rVSI, peak shift, and the arterial dominance score had higher specificity $(67.2 \%-$ $74.1 \%)$. Angiogenesis and vascular recruitment may consist of a large number of small-caliber vessels or a small number of large-caliber vessels. ${ }^{28}$ While rCBV may detect increased vascularity, it is unable to discriminate the composition of vessels from these potential circumstances. ${ }^{8}$ Because peak shift and arterial dominance scores were able to provide insight into the vascular composition and its spatial distribution, VAI parameters exhibited higher specificity in detecting PP compared with rCBV. 
Alternative approaches of vascular analysis exist, including phase-sensitive and susceptibility-weighted imaging techniques with improved detection of microvasculature and iron-containing hemorrhagic foci. In patients with posttreatment glioblastoma, a previous study showed that the increase in the percentage of hypointensity on susceptibility imaging was higher in responders than in nonresponders, and nonresponders showed a sparse amount of hypointense signal in the area of contrast enhancement. ${ }^{29}$ This finding revealed that radiation-induced damage to microvasculature resulted in the formation of microbleeds on susceptibility imaging. ${ }^{29,30}$ However, chronic hemorrhage also appears as hypointensity, and differentiation of arterial- or venous-type vasculature may not be feasible with susceptibility imaging.

This study has several limitations. First, the diagnostic performance of rCBV in our study was lower than previously reported in differentiating tumor progression from treatment-related change. This finding may be due to low rCBV values extracted from the conventional DSC MR imaging protocol with a flip angle of $35^{\circ}$, which is lower than that proposed by American Society of Functional Neuroradiology recommendations. ${ }^{31}$ The use of a lower flip angle may result in relatively small CBV from a low signal-tonoise ratio and low baseline signal ${ }^{32}$ and may undermine its discriminatory power between the 2 conditions. Previous studies ${ }^{33,34}$ quantified perfusion imaging metrics with histopathologic tumor fraction and yielded a higher diagnostic performance from active tumor portion. Because posttreatment glioblastoma exhibits spatial heterogeneity from admixed treatment effect and tumor cells, voxelwise quantification to measure distributions and parametric maps will be a preferred approach in imaging-based analysis.

In this study, we evaluated spatial heterogeneity using the arterial dominance score, but the measurement was qualitative, and future study with quantitative longitudinal assessment is deemed necessary. Second, the VAI sequence provides a limited scan range compared with conventional DSC MR imaging due to the need for an SE readout with long TEs and requires a double-dose injection of contrast material. However, the recent development of using the combined spin- and gradient-echo-based MR imaging ${ }^{35}$ allows whole-brain coverage and acquisition of robust CBV without a preload in addition to VAI. Technical updates are necessary in our protocol, though double-dose perfusion studies have been performed for $>20$ years and were acknowledged by the consensus recommendations of the American Society of Functional Neuroradiology ${ }^{31}$ and Jumpstarting Brain Tumor Drug Development Coalition Imaging Standardization Steering Committee. ${ }^{36}$

\section{CONCLUSIONS}

Vessel architectural imaging significantly improved the diagnostic performance of rCBV by demonstrating venous dominance and a tendency toward larger vessel size in early tumor progression and may serve as an early identifier of disease progression in posttreatment glioblastoma.

\section{ACKNOWLEDGMENT}

The authors thank Jeong Hoon Kim (J.H.K.) for the contribution in establishing final diagnoses of pseudoprogression and early tumor progression by clinicoradiologic consensus.
Disclosures: Kyrre Emblem-RELATED: Grant: the European Research Council; South-Eastern Norway Regional Health Authority; the Research Council of Norway, Comments: This work received funding from grant 261984, the SouthEastern Norway Regional Health Authority grants 2017073 and 2013069, and the European Research Council under the European Union's Horizon 2020 Program (European Research Council Grant agreement No. 758657-ImPRESS); UNRELATED: Patents (Planned, Pending or Issued): NordicNeuroLab AS, Comments: intellectual property rights.* Ho Sung Kim-UNRELATED: Employment: Asan Medical Center. Atle Bjørnerud_UNRELATED: Grant: This work received funding from the Research Council of Norway grant 191088/F20.* Consultancy: NordicNeuroLab AS; Stock/Stock Options: NordicNeuroLab AS. *Money paid to the institution.

\section{REFERENCES}

1. Kruser TJ, Mehta MP, Robins HI. Pseudoprogression after glioma therapy: a comprehensive review. Expert Rev Neurother 2013;13: 389-403 CrossRef Medline

2. Jensen RL. Brain tumor hypoxia: tumorigenesis, angiogenesis, imaging, pseudoprogression, and as a therapeutic target. J Neurooncol 2009;92:317-35 CrossRef Medline

3. New P. Radiation injury to the nervous system. Curr Opin Neurol 2001;14:725-34 CrossRef Medline

4. Barajas RF Jr, Chang JS, Segal MR, et al. Differentiation of recurrent glioblastoma multiforme from radiation necrosis after external beam radiation therapy with dynamic susceptibility-weighted contrast-enhanced perfusion MR imaging. Radiology 2009;253:486-96 CrossRef Medline

5. Kong DS, Kim ST, Kim EH, et al. Diagnostic dilemma of pseudoprogression in the treatment of newly diagnosed glioblastomas: the role of assessing relative cerebral blood flow volume and oxygen-6-methylguanine-DNA methyltransferase promoter methylation status. AJNR Am J Neuroradiol 2011;32:382-87 CrossRef Medline

6. Suh $\mathrm{CH}$, Kim HS, Jung SC, et al. Multiparametric MRI as a potential surrogate endpoint for decision-making in early treatment response following concurrent chemoradiotherapy in patients with newly diagnosed glioblastoma: a systematic review and metaanalysis. Eur Radiol 2018;28:2628-38 CrossRef Medline

7. Patel P, Baradaran H, Delgado D, et al. MR perfusion-weighted imaging in the evaluation of high-grade gliomas after treatment: a systematic review and meta-analysis. Neuro Oncol 2017;19:118-27 CrossRef Medline

8. Chakhoyan A, Yao J, Leu K, et al. Validation of vessel size imaging (VSI) in high-grade human gliomas using magnetic resonance imaging, image-guided biopsies, and quantitative immunohistochemistry. Sci Rep 2019;9:2846 CrossRef Medline

9. Emblem KE, Mouridsen K, Bjornerud A, et al. Vessel architectural imaging identifies cancer patient responders to anti-angiogenic therapy. Nat Med 2013;19:1178-83 CrossRef Medline

10. Dennie J, Mandeville JB, Boxerman JL, et al. NMR imaging of changes in vascular morphology due to tumor angiogenesis. Magn Reson Med 1998;40:793-99 CrossRef Medline

11. Boxerman JL, Hamberg LM, Rosen BR, et al. MR contrast due to intravascular magnetic susceptibility perturbations. Magn Reson Med 1995;34:555-66 CrossRef Medline

12. Kiselev VG, Strecker R, Ziyeh S, et al. Vessel size imaging in humans. Magn Reson Med 2005;53:553-63 CrossRef Medline

13. Louis DN, Perry A, Reifenberger G, et al. The 2016 World Health Organization Classification of Tumors of the Central Nervous System: a summary. Acta Neuropathol 2016;131:803-20 CrossRef Medline

14. Stupp R, Mason WP, van den Bent MJ, et al; European Organisation for Research and Treatment of Cancer Brain Tumor and Radiotherapy Groups; National Cancer Institute of Canada Clinical Trials Group. Radiotherapy plus concomitant and adjuvant temozolomide for glioblastoma. N Engl J Med 2005;352: 987-96 CrossRef Medline

15. Wen PY, Macdonald DR, Reardon DA, et al. Updated Response Assessment Criteria for High-Grade Gliomas: Response Assessment 
in Neuro-Oncology Working Group. J Clin Oncol 2010;28:1963-72 CrossRef Medline

16. Weisskoff RM, Boxerman JL, Sorensen AG, et al. Simultaneous blood volume and permeabilitymapping using a single Gd-based contrastinjection. In: Proceedings of the Society of Magnetic Resonance, second annual meeting, San Francisco, CA, USA. August 6-12, 1994

17. Boxerman JL, Schmainda KM, Weisskoff RM. Relative cerebral blood volume maps corrected for contrast agent extravasation significantly correlate with glioma tumor grade, whereas uncorrected maps do not. AJNR Am J Neuroradiol 2006;27:859-67 Medline

18. Stadlbauer A, Zimmermann M, Oberndorfer S, et al. Vascular hysteresis loops and vascular architecture mapping in patients with glioblastoma treated with antiangiogenic therapy. Sci Rep 2017; 7:8508 CrossRef Medline

19. Xu C, Kiselev VG, Moller HE, et al. Dynamic hysteresis between gradient echo and spin echo attenuations in dynamic susceptibility contrast imaging. Magn Reson Med 2013;69:981-91 CrossRef Medline

20. Digernes I, Bjornerud A, Vatnehol SA, et al. A theoretical framework for determining cerebral vascular function and heterogeneity from dynamic susceptibility contrast MRI. J Cereb Blood Flow Metab 2017;37:2237-48 CrossRef Medline

21. Madsen MT. A simplified formulation of the gamma variate function. Phys Med Biol 1992;37:1597-1600 CrossRef

22. Press WH, Flannery BP, Teukolsky SA, et al. Numerical Recipes in C: The Art of Scientific Computing. Cambridge University Press; 1988

23. Han K, Song K, Choi BW. How to develop, validate, and compare clinical prediction models involving radiological parameters: study design and statistical methods. Korean J Radiol 2016;17:339-50 CrossRef Medline

24. Pries AR, Hopfner M, Le Noble F, et al. The shunt problem: control of functional shunting in normal and tumour vasculature. Nat Rev Cancer 2010;10:587-93 CrossRef Medline

25. Mathivet T, Bouleti C, Van Woensel M, et al. Dynamic stroma reorganization drives blood vessel dysmorphia during glioma growth. Embo Mol Med 2017;9:1629-45 CrossRef Medline

26. Stadlbauer A, Eyupoglu I, Buchfelder M, et al. Vascular architecture mapping for early detection of glioblastoma recurrence. Neurosurg Focus 2019;47:E14 CrossRef Medline
27. van Dijken BR, van Laar PJ, Holtman GA, et al. Diagnostic accuracy of magnetic resonance imaging techniques for treatment response evaluation in patients with high-grade glioma: a systematic review and meta-analysis. Eur Radiol 2017;27:4129-44 CrossRef Medline

28. Nakada $M$, Kita $D$, Watanabe $T$, et al. Aberrant signaling pathways in glioma. Cancers (Basel) 2011;3:3242-78 CrossRef Medline

29. Lupo JM, Essock-Burns E, Molinaro AM, et al. Using susceptibilityweighted imaging to determine response to combined anti-angiogenic, cytotoxic, and radiation therapy in patients with glioblastoma multiforme. Neuro Oncol 2013;15:480-89 CrossRef Medline

30. Lupo JM, Chuang CF, Chang SM, et al. 7-Tesla susceptibilityweighted imaging to assess the effects of radiotherapy on normalappearing brain in patients with glioma. Int $J$ Radiat Oncol Biol Phys 2012;82:e493-500 CrossRef Medline

31. Welker K, Boxerman J, Kalnin A, et al; American Society of Functional Neuroradiology MR Perfusion Standards and Practice Subcommittee of the ASFNR Clinical Practice Committee. ASFNR recommendations for clinical performance of MR dynamic susceptibility contrast perfusion imaging of the brain. AJNR Am J Neuroradiol 2015;36:E41-51 CrossRef Medline

32. Cha S, Knopp EA, Johnson G, et al. Intracranial mass lesions: dynamic contrast-enhanced susceptibility-weighted echo-planar perfusion MR imaging. Radiology 2002;223:11-29 CrossRef Medline

33. Hu LS, Eschbacher JM, Heiserman JE, et al. Reevaluating the imaging definition of tumor progression: perfusion MRI quantifies recurrent glioblastoma tumor fraction, pseudoprogression, and radiation necrosis to predict survival. Neuro Oncol 2012;14:919-30 CrossRef Medline

34. Prah MA, Al-Gizawiy MM, Mueller WM, et al. Spatial discrimination of glioblastoma and treatment effect with histologically-validated perfusion and diffusion magnetic resonance imaging metrics. J Neurooncol 2018;136:13-21 CrossRef

35. Zhang K, Yun SD, Triphan SM, et al. Vessel architecture imaging using multiband gradient-echo/spin-echo EPI. PLos One 2019;14: e0220939 CrossRef Medline

36. Boxerman JL, Quarles CC, Hu LS, et al; Jumpstarting Brain Tumor Drug Development Coalition Imaging Standardization Steering Committee. Consensus recommendations for a dynamic susceptibility contrast MRI protocol for use in high-grade gliomas. Neuro Oncol 2020;22:1262-75 CrossRef Medline 